\title{
DNA capture into the ClyA nanopore: diffusion-limited versus reaction-limited processes
}

\author{
Stefanos K Nomidis ${ }^{1,2} \oplus$, Jef Hooyberghs ${ }^{2}$, Giovanni Maglia ${ }^{3}$ \\ and Enrico Carlon ${ }^{1}$ (1)
}

${ }^{1}$ KU Leuven, Institute for Theoretical Physics, Celestijnenlaan 200D, 3001 Leuven, Belgium

${ }^{2}$ Flemish Institute for Technological Research (VITO), Boeretang 200, B-2400 Mol, Belgium

3 Chemical Biology, Groningen Biomolecular Sciences \& Biotechnology Institute, University of Groningen, Nijenborgh 7, 9747 AG Groningen, The Netherlands

E-mail: enrico.carlon@kuleuven.be

Received 1 March 2018, revised 8 June 2018

Accepted for publication 12 June 2018

Published 29 June 2018

\begin{abstract}
The capture and translocation of biomolecules through nanometer-scale pores are processes with a potentially large number of applications, and hence they have been intensively studied in recent years. The aim of this paper is to review existing models of the capture process by a nanopore, together with some recent experimental data of short single- and double-stranded DNA captured by the Cytolysin A (ClyA) nanopore. ClyA is a transmembrane protein of bacterial origin which has been recently engineered through site-specific mutations, to allow the translocation of double- and single-stranded DNA. A comparison between theoretical estimations and experiments suggests that for both cases the capture is a reaction-limited process. This is corroborated by the observed salt dependence of the capture rate, which we find to be in quantitative agreement with the theoretical predictions.
\end{abstract}

Keywords: transport, DNA capture, biological nanopores

(Some figures may appear in colour only in the online journal)

\section{Introduction}

Current nanopore technologies offer a large number of interesting applications for the analysis of DNA, proteins, peptides and other types of small molecules [1-5]. Such devices detect the presence of single molecules by measuring a drop in the ionic current passing through the pore. Two different types of nanopores are presently used; solid-state nanopores can be fabricated by various techniques that produce a small hole in a silicon [1] or graphene membrane [6]. The size and shape of these nanopores can be tuned during the fabrication process. Biological nanopores, on the other hand, are proteins, typically of bacterial origin, embedded within a lipid bilayer $[2,7]$. Compared to solid-state nanopores the size of biological pore proteins cannot be tuned, but they can be engineered with atomic precision by site-specific mutations $[8,9]$. The most studied biological nanopore is the alpha-hemolysin $(\alpha \mathrm{HL})$ protein, which is used in the first commercial nanopore DNA sequencer [10]. Owing to the narrow inner-pore constriction $(1.3 \mathrm{~nm})$, translocation through $\alpha \mathrm{HL}$ is restricted to singlestranded DNA (ssDNA). While nanopore DNA sequencers are based on the translocation of ssDNA, for other applications it is desirable to consider pores also allowing the translocation of double-stranded DNA (dsDNA). The electrophoretic translocation of ssDNA across biological nanopores of about $1 \mathrm{~nm}$ diameter has been extensively studied on view of its relevance to DNA sequencing. The dsDNA translocation has received less attention, primarily due to the lack of biological nanopores with a diameter large enough to allow translocation. There are, however, several applications possible for dsDNA translocation, as the detection of DNA-binding proteins [11] or of molecules that alter the double helix geometry [12]. A recent review about biological nanopore sensing and a discussion of commonly-utilized nanopores can be found in [7]. 
a)

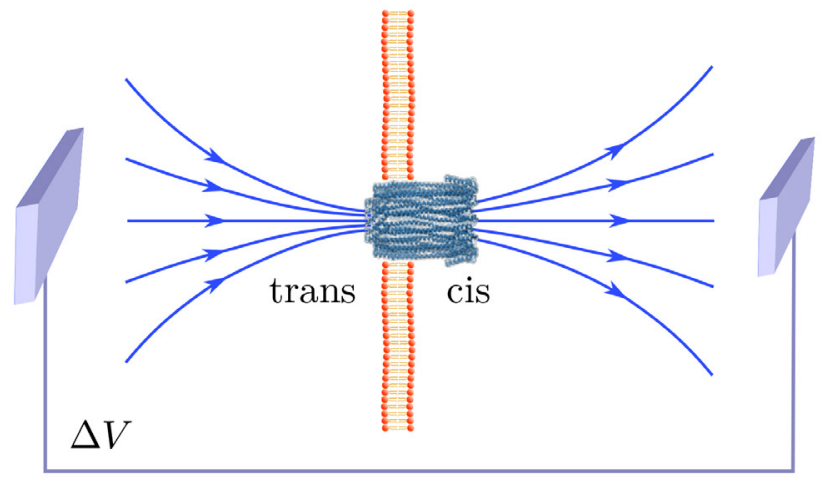

b)

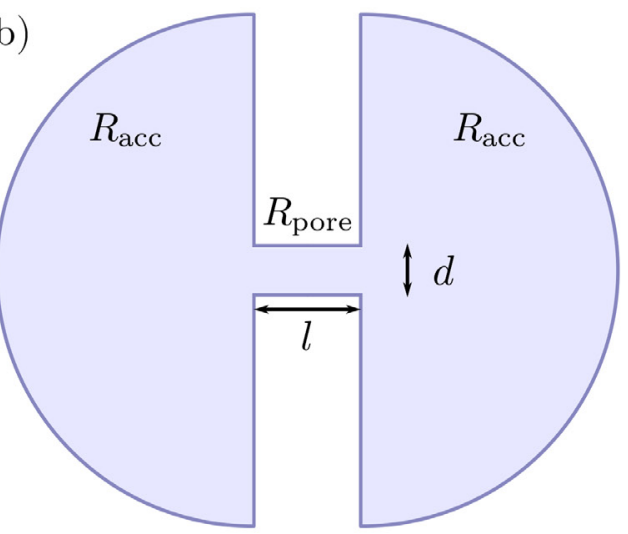

Figure 1. (a) Sketch of a typical experimental setup. A potential difference $\Delta V$ is applied between two electrodes placed at the two far sides of the membrane. The arrows indicate the direction of electric field lines. (b) Physically, the system can be viewed as a collection of three resistors in series, one corresponding to the nanopore $R_{\text {pore }}$, and two access resistances $R_{\text {acc }}$ at its sides (shaded areas). Note that the latter are assumed to extend to infinity.
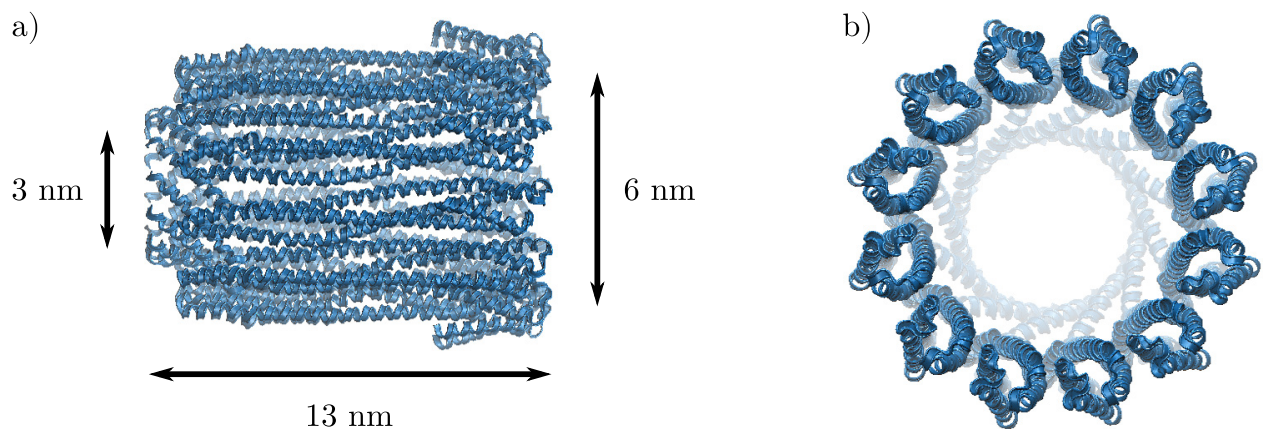

Figure 2. Side (a) and top (b) view of the ClyA nanopore (PDB: 2WCD), with the characteristic size of the narrow and wide entries shown. For the visualization of the structure we used the VMD software [16]

In this paper we analyze the capture of both ssDNA and dsDNA by Cytolysin A (ClyA), a biological nanopore which has been recently employed both for nucleic acid and protein analysis $[3,4,9,13]$. In experiments, DNA molecules are initially placed in the cis-side of the membrane. An electric field is induced by applying a potential difference between two electrodes placed at the two opposite sides of the membrane (see figure 1(a)). As a result, negatively-charged DNA molecules diffusing in the vicinity of the nanopore are attracted to the pore entry. After their eventual capture they either translocate to the trans-side, or are released back to the cis-side. Here we review the theory of the DNA capture and discuss two possible mechanisms of diffusion-limited and reactionlimited capture $[14,15]$. We compare the two mechanisms with experiments for short ssDNA and dsDNA captured by a ClyA nanopore. We show that the dependence of these rates on the ionic strength of the solution suggests that for both molecules the capture is a reaction-limited process.

\section{The ClyA nanopore}

ClyA is a toxin synthesized by several bacteria, and is employed to disrupt cellular membranes of other organisms. It is initially synthesized as a monomer, and then it spontaneously assembles into a 12-mer, cylindrically-shaped pore (figure 2). The internal diameter is about $3 \mathrm{~nm}$ at the narrower side and $6 \mathrm{~nm}$ at the wider side, while its length is $13 \mathrm{~nm}$. Although the diameter of ClyA can, in principle, fit both ssDNA and dsDNA, owing to the negative charges present in the pore lumen, translocation in the wild type ClyA can only occur in solutions of high ionic strength. For this reason ClyA mutants were recently engineered [13], that contain additional positive charges in the lumen and the wide entrance of the pore, allowing DNA translocation at physiological salt concentrations $(150 \mathrm{mM} \mathrm{NaCl})$.

\section{Modeling the DNA capture}

Figure 1(a) shows a typical experimental setup, in which a single nanopore is inserted in the lipid bilayer membrane, in contact with a $\mathrm{NaCl}$ solution. When a potential difference $\Delta V$ is applied between two electrodes, and in absence of blockages at the pore, a steady electric current pore $I$ is generated, with oppositely-charged ions flowing through the pore in opposite directions. The current can be calculated by first decomposing the system into two semi-infinite spherical shells (cis and trans side), connected with each other through a cylinder of diameter $d$ and length $l$ (nanopore). Then, treating the three regions as resistors in series (figure 1(b)) and using Ohm's law yields [14] 


$$
\Delta V=I\left(R_{\text {pore }}+2 R_{\mathrm{acc}}\right)=\frac{I}{\sigma}\left(\frac{4 l}{\pi d^{2}}+\frac{1}{d}\right),
$$

where $\sigma$ is the conductivity. We have denoted by $R_{\text {acc }}$ the resistance of each semi-infinite half sphere, known as the access resistance (the derivation can be found in [17]), and by $R_{\text {pore }}$ the electric resistance of the pore (figure 1(b)). The contribution of $R_{\text {acc }}$ becomes dominant for wider solid-state nanopores, as confirmed by experiments with nanopores of varying $d$ [18]. In the case of ClyA, which has dimensions $d=6 \mathrm{~nm}$ and $l=13 \mathrm{~nm}$, one finds $2 R_{\text {acc }} / R_{\text {pore }} \approx 0.36$. Assuming that the equipotential surfaces are semi-spherical outside the pore, one obtains the electrostatic potential [14]

$$
V(r)=\frac{I}{2 \pi \sigma r}=\frac{\Delta V}{2 \pi r}\left(\frac{4 l}{\pi d^{2}}+\frac{1}{d}\right)^{-1} \equiv \Delta V \frac{\tilde{d}}{r},
$$

where we set the potential to zero at the electrode $r \rightarrow \infty$ and defined the characteristic length

$$
\widetilde{d} \equiv \frac{1}{2 \pi}\left(\frac{4 l}{\pi d^{2}}+\frac{1}{d}\right)^{-1},
$$

which depends only on the geometry of the pore (for the case of ClyA one finds $\widetilde{d}=0.25 \mathrm{~nm}$ ). The DNA molecule performs a drift-diffusive motion in the potential (equation (2)) until it reaches the close vicinity of the pore. There, it is either directly translocated to the other side of the membrane, corresponding to a diffusion-limited case, or it encounters an additional free energy barrier that needs to overcome for a successful translocation. If the barrier is large compared to the thermal energy $k_{\mathrm{B}} T$ and the attractive electrostatic potential, it will dominate the capture kinetics, and the process becomes reaction-limited. We will discuss these two cases separately, following closely the theory developed in [14].

\subsection{Diffusion-limited capture}

In spite of its high complexity, far from the pore the problem becomes spherically symmetric (see equation (3)), and DNA can be treated as a charged point particle. Let us consider a collection of such diffusing particles characterized by a concentration $c(\vec{r}, t)$ and subject to an electrophoretic force, given by a radial potential $V(r)$. The continuity equation in spherical coordinates reads

$$
\frac{\partial c}{\partial t}=-\frac{1}{r^{2}} \frac{\partial}{\partial r}\left[r^{2} j(r)\right]
$$

where the radial current density contains the contribution from diffusion and electrophoretic drift

$$
j(r)=-D \frac{\partial c}{\partial r}+\mu c \frac{\partial V}{\partial r}
$$

Combining equations (4) and (5) one obtains the drift-diffusion equation, with $D$ and $\mu$ the diffusion coefficient and the electric mobility, respectively. Note that the two terms in equation (5) enter with a different sign because DNA is negatively charged, and by convention $\mu>0$. It should be stressed that the Einstein relation does not hold for free electrophoresis of DNA [19], i.e. $D \neq \mu k_{\mathrm{B}} T$. This arises from the fact that a free DNA in solution is accompanied by a collection of counterions, while the application of an electric field pushes the two in opposite directions. This leads to different typical molecular configurations, hence the Einstein relation breaks down.

The stationary solution of equation (4) is obtained by setting $\partial c / \partial t=0$, corresponding to constant $r^{2} j(r)$. For the $1 / r$ potential of equation (2) one finds [14]

$$
c(r)=c_{0} \frac{1-\mathrm{e}^{-r^{*}(1 / R-1 / r)}}{1-\mathrm{e}^{-r^{*} / R}},
$$

where $c_{0}$ is the bulk concentration and $r^{*}$ a characteristic length given by

$$
r^{*} \equiv \frac{\mu \widetilde{d} \Delta V}{D} .
$$

For the derivation of equation (6) we used as boundary conditions $\lim _{r \rightarrow \infty} c(r)=c_{0}$ and $c(R)=0$, with $R$ a microscopic distance of the order of the pore size. From equation (6) one can estimate the capture rate, which is equal to the number of particles per unit time reaching the absorbing boundary at $r=R$. This is obtained by integrating the current density on a half-spherical shell of radius $r$ [14]

$$
k_{\mathrm{on}}=2 \pi r^{2} j(r)=\frac{2 \pi D r^{*} c_{0}}{1-\mathrm{e}^{-r^{*} / R}} \approx 2 \pi D r^{*} c_{0},
$$

where we have used $r^{*} \gg R$, which is a valid approximation for typical systems [13]. Here $r^{*}$ can be interpreted as the distance at which the DNA is irreversibly captured by the pore [14], and increases with the applied potential and the electrophoretic mobility (see equation (7)). Equation (8) is identical to the Smoluchowski diffusion-limited reaction rate for a free diffusing particle absorbed by a sphere of radius $r^{*}$, with $2 \pi$ instead of $4 \pi$ due to the semi-infinite geometry [14].

\subsection{Reaction-limited capture}

In a reaction-limited capture the actual translocation takes place once DNA overcomes an additional barrier at the pore entry. Rowghanian and Grosberg discussed this type of process, which we review here. Let us consider a drift-diffusion model with an additional short-range repulsive potential $U(r)$, i.e. nonvanishing only in the close vicinity of the pore. The radial current density is then given by

$$
j(r)=-D \frac{\partial c}{\partial r}+\mu c \frac{\partial V}{\partial r}-\tilde{\mu} c \frac{\partial U}{\partial r},
$$

where we distinguish between the electrophoretic mobility $\mu$ and a mobility $\widetilde{\mu}$ connected to other external forces [20]. Although the former does not satisfy the Einstein relation, the latter does [21], i.e. $D=\widetilde{\mu} k_{\mathrm{B}} T$. Thus, one can rewrite the current as

$$
j(r)=-D\left(\frac{\partial c}{\partial r}+\frac{c}{k_{\mathrm{B}} T} \frac{\partial \Phi(r)}{\partial r}\right),
$$




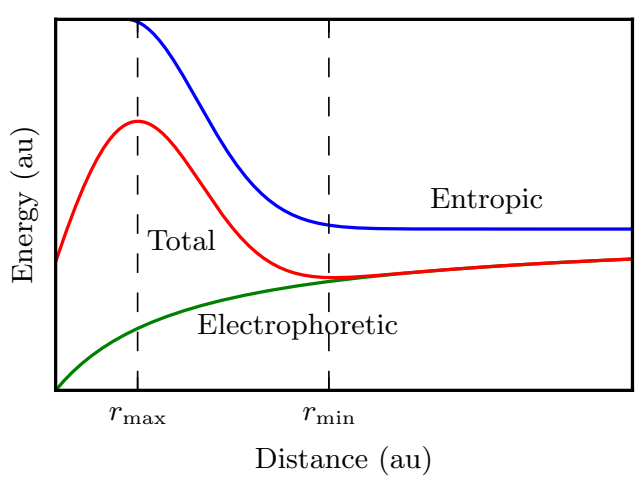

Figure 3. Schematic plot of the effective potential in the vicinity of the pore for a reaction-limited capture. In addition to the contribution of the long-ranged, attractive electrophoretic force, a substantial short-ranged repulsive force is expected to arise due to steric effects for DNA threading into the pore $[14,22]$. The sum of the two potentials has a minimum in $r_{\min }$ and a maximum in $r_{\max }$.

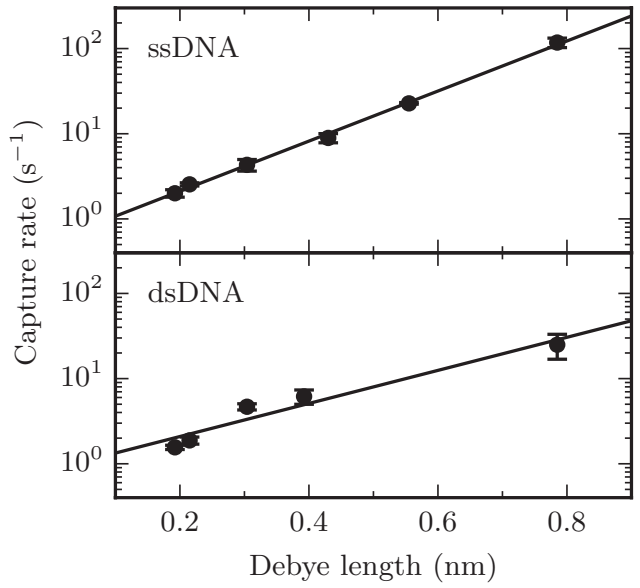

Figure 4. Capture rate of ssDNA (top) and dsDNA (bottom) by a ClyA nanopore at varying ionic strength of the solution (data from [13]). The data are plotted as a function of the Debye length $\lambda_{\mathrm{D}}$, which is related to the salt concentration in solution $n_{0}$ as $\lambda_{\mathrm{D}}=\sqrt{k_{\mathrm{B}} T / 4 \pi \mathrm{e}^{2} n_{0}}$. In the experiments the $\mathrm{NaCl}$ concentration was varied in the range $[0.15,2.5] \mathrm{M}$, corresponding to a range $[0.2,0.8] \mathrm{nm}$ for the Debye length. The solid lines were obtained from least-squares fitting of an exponential function $k_{\text {on }} \sim \exp \left(\lambda_{\mathrm{D}} / s\right)$, which yielded $s=0.15 \mathrm{~nm}$ (ssDNA) and $s=0.22 \mathrm{~nm}(\mathrm{dsDNA})$.

with

$$
\Phi(r) \equiv U(r)-\frac{\mu}{\widetilde{\mu}} V(r) .
$$

Thus, the dynamics is described by a drift-diffusion equation in the effective potential $\Phi(r)$. The electrophoretic contribution to the force is attractive, while $U(r)$ is short-range and repulsive and expected to originate from the steric hindrance of the DNA threading into the pore [14, 22] (figure 3). To initiate translocation the molecules have to overcome a barrier $\Delta \Phi=\Phi\left(r_{\max }\right)-\Phi\left(r_{\min }\right)$, where $r_{\max }$ and $r_{\min }$ are the positions of the maximum and minimum of $\Phi$, respectively, located in the vicinity of the pore (figure 3 ). If the capture process is reaction-limited (i.e. $\Delta \Phi \gg k_{\mathrm{B}} T$ ), we expect that the rate will be given by

$$
k_{\mathrm{on}}=\omega \mathrm{e}^{-\Delta \Phi / k_{\mathrm{B}} T},
$$

where $\omega$ is a characteristic rate constant.

\section{Experiments}

Having reviewed the existing theory of DNA capture by nanopore, we will now apply it to the experimental data of [13]. In that study the capture rates of both ssDNA and dsDNA by the ClyA nanopore were measured as a function of the ionic strength of the solution. In what follows we will show that the experimental data in both cases (shown in figure 4) are not in line with the theory of diffusion-limited capture. Instead, they seem to exhibit the exponential dependence predicted by equation (12), suggesting that it is more likely a reaction-limited process. Finally, we will show that the fitted exponent is in line, at least in the order of magnitude, with the theoretical predictions, further reinforcing our argument.

Since both theories involve the electrophoretic mobility $\mu$ of DNA, we will first estimate its value. Assuming that DNA is a cylinder of diameter $b$ with an effective charge per length equal to $-g \alpha e / a$ (with $a$ the separation between successive phosphate groups), [14] estimates the electrophoretic mobility as

$$
\mu=\frac{g \alpha e \lambda_{\mathrm{D}}}{\eta \pi a b},
$$

where $\eta=10^{-3} \mathrm{~kg} \mathrm{~m}^{-1} \mathrm{~s}^{-1}$ is the water viscosity, and $\alpha<1$ is a numerical factor. The latter takes into account that counterions are bound to the phosphate charges, rendering the effective charge of DNA smaller than the bare one. Finally, one should use $g=1$ and $g=2$ for ssDNA and dsDNA, respectively. Since this quantity enters in both theories, we will calculate $\mu \Delta V$ for both ssDNA and dsDNA. Using $a b \approx 0.68$ $\mathrm{nm}^{2}$ and $\Delta V=70 \mathrm{mV}$ we obtain ${ }^{4}$

$$
\mu \Delta V \approx 5.2 g \lambda_{\mathrm{D}} \mathrm{m} \mathrm{s}^{-1},
$$

where for simplicity we have taken $\alpha=1$. Thus, the electrophoretic mobilities of ssDNA and dsDNA are found to be quite similar.

We will first test whether the data can be described by the theory of diffusion-limited capture. Combining equations (7) and (8) yields $k_{\mathrm{on}}=2 \pi \mu \Delta V \widetilde{d} c_{0}$, so using the experimental concentration $c_{0}=1 \mu \mathrm{M}$, the characteristic length $\widetilde{d}=0.25$ $\mathrm{nm}$ (see discussion below equation (3)) and equation (14) yields

$$
k_{\text {on }}=2.5 \mathrm{~g} \times 10^{3} \mathrm{~s}^{-1},
$$

where we have used a representative value $\lambda_{\mathrm{D}}=0.5 \mathrm{~nm}$ for the Debye length. A comparison with the experimental data of figure 4 indicates that this result overestimates the capture rates by two orders of magnitude. Note that some of the phosphate DNA charges can be bound to counterions, leading to

\footnotetext{
${ }^{4}$ For dsDNA it is $a=0.34 \mathrm{~nm}$ and $b=2 \mathrm{~nm}$, while for ssDNA one has $a=0.68 \mathrm{~nm}$ and $b \approx 1 \mathrm{~nm}[23]$.
} 
$\alpha<1$. However, to reconcile the data with diffusion-limited capture, one would need a very small value of $\alpha \sim 10^{-2}$, which is unlikely. In addition, the data are not consistent with a linear dependence on $\lambda_{\mathrm{D}}$, as expected from a diffusion-limited process $\left(k_{\text {on }} \sim \mu \sim \lambda_{\mathrm{D}}\right)$. We, thus, conclude that the capture of both ssDNA and dsDNA is not diffusion-limited ${ }^{5}$. This is in agreement with measurements for dsDNA of comparable size captured by solid-state nanopores [24].

Having excluded a diffusion-limited capture, let us now test the other limiting case, that of a reaction-limited process. Combining equations (11) and (12) one obtains

$$
k_{\text {on }} \sim \exp \left[\frac{\mu}{\widetilde{\mu} k_{\mathrm{B}} T}\left(V_{\max }-V_{\min }\right)\right],
$$

where we have defined $V_{\max } \equiv V\left(r_{\max }\right)$ and $V_{\min } \equiv V\left(r_{\min }\right)$ the maximum and minimum values of the electrophoretic potential. Note that we have omitted the entropic part $U\left(r_{\max }\right)-U\left(r_{\min }\right)$ and numerical prefactors in the previous expression, as we focus on the dependence of $k_{\text {on }}$ on the Debye length, which enters in the problem via the electophoretic mobility $\mu$ (equation (13)). The omitted factors are hence $\lambda_{\mathrm{D}}$-independent. In combination with equation (13), this relation implies an exponential dependence of the capture rate on the Debye length. This is indeed the observed trend of the experimental data, as seen in figure 4 . For a more quantitative comparison, we get from equation (2)

$$
V_{\max }-V_{\min }=\Delta V \widetilde{d}\left(\frac{1}{r_{\max }}-\frac{1}{r_{\min }}\right) \equiv \Delta V \frac{\widetilde{d}}{\widetilde{r}},
$$

where $\widetilde{r}$ is a characteristic length, and is expected to be comparable to the pore diameter, i.e. $\widetilde{r} \sim d=6 \mathrm{~nm}$. Combining this with equation (16) yields

$$
k_{\mathrm{on}} \sim \exp \left(\frac{\mu \Delta V}{\widetilde{\mu} k_{\mathrm{B}} T} \frac{\widetilde{d}}{d}\right) .
$$

The only missing element is the determination of the mechanical mobility $\widetilde{\mu}$. For this purpose one may use Stokes' law, which gives

$$
\widetilde{\mu} k_{\mathrm{B}} T=\frac{k_{\mathrm{B}} T}{6 \pi \eta R_{\mathrm{H}}} \approx \frac{2.2 \times 10^{-19}}{R_{\mathrm{H}}} \mathrm{m}^{3} \mathrm{~s}^{-1},
$$

where $R_{\mathrm{H}}$ is the hydrodynamic radius. Combining this with equations (14) and (18), and using once more $\widetilde{d}=0.25 \mathrm{~nm}$ and $d=6 \mathrm{~nm}$, gives $k_{\mathrm{on}} \sim \exp \left(0.98 g \lambda_{\mathrm{D}} R_{\mathrm{H}} \mathrm{nm}^{-2}\right) \equiv \exp \left(\lambda_{\mathrm{D}} / s\right)$, where

$$
s=\frac{1 \mathrm{~nm}}{0.98 g R_{\mathrm{H}}},
$$

is a parameter that can be fitted to the experimental data (see figure 4). Since the contour length $L=62 \mathrm{~nm}$ of ssDNA is much larger than its persistence length $l_{\mathrm{p}}=2.2 \mathrm{~nm}$, and if we neglect excluded-volume effects, we can approximate it as a

\footnotetext{
${ }^{5}$ Franceschini et al [13] suggested for ssDNA a reaction-limited capture and for dsDNA a diffusion-limited capture. Though building on the same theory as here, the latter conclusion was based on an erroneous arithmetic calculation, which underestimated $k_{\text {on }}$ for the diffusion-limited case.
}

sphere of radius $\sqrt{l_{\mathrm{p}} L / 3}$ (radius of gyration). Using this for the estimation of its hydrodynamic radius gives $R_{\mathrm{H}} \approx 7 \mathrm{~nm}$, from which we find $s \approx 0.15 \mathrm{~nm}$. In the case of dsDNA, the contour length $L=31 \mathrm{~nm}$ is lower than its persistence length $l_{\mathrm{p}}=45 \mathrm{~nm}$, suggesting it behaves more like a rigid rod. If we, once more, approximate it as a cylinder of diameter $b$, and use the results of [25], we obtain $R_{\mathrm{H}} \approx 5 \mathrm{~nm}$. Plugging this in equation (20) yields $s \approx 0.10 \mathrm{~nm}$. These results are in a good agreement with fits of the experimental data (figure 4), which yielded the values $s=0.15 \mathrm{~nm}$ and $s=0.22 \mathrm{~nm}$ for ssDNA and dsDNA, respectively, despite the simplicity of the theory. The agreement further corroborates the validity of the reaction-limited capture scenario.

To support this conclusion we, finally, provide a simple estimate of the entropic barrier for the dsDNA translocation, modeling again the latter as a rigid rod of length $L=31 \mathrm{~nm}$. When the rod is sufficiently far from the pore, its orientation can explore a full solid angle $\Omega_{\text {bulk }}=4 \pi$. When, however, the rod end enters the pore with its center aligned to the pore axis, the solid angle reduces to

$$
\Omega_{\text {pore }}=4 \pi\left(1-\sqrt{1-\frac{d^{2}}{L^{2}}}\right) \approx \frac{2 \pi d^{2}}{L^{2}},
$$

where $d=6 \mathrm{~nm}$ is the pore diameter. In the last equality we have also used $d \ll L$. The change in the orientational entropy is $\Delta S_{\text {or. }}=-k_{\mathrm{B}} \log \left(\Omega_{\text {pore }} / \Omega_{\text {bulk }}\right)$. Assuming that the electric field is almost constant close to the pore, and that the predominant contribution to the free energy comes from the orientational entropy loss, we estimate

$$
\Delta \Phi \approx k_{\mathrm{B}} T \Delta S_{\text {or. }} \approx k_{\mathrm{B}} T \log \left(\frac{2 L^{2}}{d^{2}}\right) \approx 4 k_{\mathrm{B}} T,
$$

which, although obtained from a very simplified calculation, suggests that dsDNA capture is a reaction-limited process. A theory for the barrier-limited capture of long DNA coils, which should be appropriate for ssDNA, was developed in $[15,21]$. In that case one should include a conformational entropy loss for placing the end of the DNA chain close to the pore entrance. The theory contains energetic parameters that are difficult to estimate, whereas estimating the rod orientational entropy (dsDNA) is straightforward.

\section{Conclusion}

We have reviewed two basic mechanisms of DNA capture by a nanopore: the diffusion-limited and the reaction-limited capture. The theoretical description of these mechanisms was developed in [14, 22, 24], and these ideas were tested in translocation experiments through solid-state nanopores with dsDNA sequences ranging from 800 to 50000 base pairs [24]. The shortest length dsDNA (up to 8000 base pairs) showed a reaction-limited capture, characterized by an exponential growth of the capture rate $k_{\text {on }}$ with the sequence length. A second regime, for sequences longer than 10000 base pairs, was found to be consistent with a diffusion-limited capture, in which $k_{\text {on }}$ is independent of the sequence length [24]. Overall, 
solid-state nanopore experiments [24] were found to be in agreement with the theoretical framework of dsDNA capture.

Here we tested the theory in a set of experiments with ClyA, a biological nanopore recently engineered to allow translocation of both ssDNA and dsDNA at physiological salt concentrations [13]. The experiments involved short ssDNA and dsDNA sequences (90 nucleotides and base pairs, respectively), and were performed at varying salt concentration [13]. Diffusionlimited capture rates estimated for a nanopore with the ClyA size were shown to be much higher than experimental measurements for both ssDNA and dsDNA, suggesting for both a reaction-limited capture (this corrects the erroneous conclusion in [13]) Our analysis showed that the experiments are in quantitative agreement with the theory, which predicts an exponential dependence $k_{\text {on }} \sim \exp \left(\lambda_{\mathrm{D}} / s\right)$ on the Debye length $\lambda_{\mathrm{D}}$, with the prefactors determined by the local properties of the barrier. The theoretical estimates for the characteristic length $s$ for both ssDNA and dsDNA are in agreement with fits to the experimental data, confirming the validity of the modeling approach. A consistent picture thus emerges for the capture mechanism of DNA from ClyA nanopore. A reaction-limited capture was also found to be in agreement with the results of [24], and with other studies of ssDNA capture into $\alpha \mathrm{HL}$ nanopores [26-28]. Still, it would be desirable to have more insight on the nature of the barrier. A question, which could be addressed by additional experiments or computer simulations of the capture mechanism, similar to those of $[29,30]$.

\section{Acknowledgment}

SN acknowledges financial support from the Research Funds Flanders (FWO Vlaanderen) grant VITO-FWO 11.59.71.7.

\section{ORCID iDs}

Stefanos K Nomidis (1) http://orcid.org/0000-0002-4635-8433

Enrico Carlon () https://orcid.org/0000-0001-8266-1096

\section{References}

[1] Dekker C 2007 Nat. Nanotechnol. 2209
[2] Venkatesan B M and Bashir R 2011 Nat. Nanotechnol. 6615

[3] Soskine M, Biesemans A, Moeyaert B, Cheley S, Bayley H and Maglia G 2012 Nano Lett. 12 4895-900

[4] Soskine M, Biesemans A and Maglia G 2015 J. Am. Chem. Soc. $1375793-7$

[5] Huang G, Willems K, Soskine M, Wloka C and Maglia G 2017 Nat. Commun. 8935

[6] Heerema S J and Dekker C 2016 Nat. Nanotechnol. 11127

[7] Shi W, Friedman A K and Baker L A 2016 Anal. Chem. 89 157-88

[8] Ayub M and Bayley H 2016 Curr. Opin. Chem. Biol. 34 117-26

[9] Soskine M, Biesemans A, De Maeyer M and Maglia G 2013 J. Am. Chem. Soc. 135 13456-63

[10] Bayley H 2015 Clin. Chem. 61 25-31

[11] Celaya G, Perales-Calvo J, Muga A, Moro F and Rodriguez-Larrea D 2017 ACS Nano 11 5815-25

[12] Yao F, Duan J, Wang Y, Zhang Y, Guo Y, Guo H and Kang X 2014 Anal. Chem. 87 338-42

[13] Franceschini L, Brouns T, Willems K, Carlon E and Maglia G 2016 ACS Nano 10 8394-402

[14] Grosberg A Y and Rabin Y 2010 J. Chem. Phys. 133165102

[15] Rowghanian P and Grosberg A Y 2013 Phys. Rev. E 87042722

[16] Humphrey W, Dalke A and Schulten K 1996 J. Mol. Graph. 1433-8

[17] Hall J E 1975 J. Gen. Physiol. 66 531-2

[18] Kowalczyk S W, Grosberg A Y, Rabin Y and Dekker C 2011 Nanotechnology 22315101

[19] Nkodo A E, Garnier J M, Tinland B, Ren H, Desruisseaux C, McCormick L C, Drouin G and Slater G W 2001 Electrophoresis 22 2424-32

[20] Long D, Viovy J L and Ajdari A 1996 Phys. Rev. Lett. 76 3858-61

[21] Rowghanian P and Grosberg A Y 2013 Phys. Rev. E 87042723

[22] Muthukumar M 2010 J. Chem. Phys. 132 05B605

[23] Chi Q, Wang G and Jiang J 2013 Physica A 392 1072-9

[24] Wanunu M, Morrison W, Rabin Y, Grosberg A Y and Meller A 2010 Nat. Nanotechnol. 5160

[25] Hansen S 2004 J. Chem. Phys. 121 9111-5

[26] Henrickson S E, Misakian M, Robertson B and Kasianowicz J J 2000 Phys. Rev. Lett. 853057

[27] Meller A and Branton D 2002 Electrophoresis 23 2583-91

[28] Meller A 2003 J. Phys.: Condens. Matter 15 R581

[29] Farahpour F, Maleknejad A, Varnik F and Ejtehadi M R 2013 Soft Matter 9 2750-9

[30] Farahpour F, Ejtehadi M R and Varnik F 2014 Int. J. Mod. Phys. C 251441010 